FOGAÇA, F.H.S. et al. Oxidação lipídica em filés de tambaqui (Colossoma macropomum) defumados com alecrim (Rosmarinus officinalis). PUBVET, Londrina, V. 8, N. 10, Ed. 259, Art. 1717, Maio, 2014.

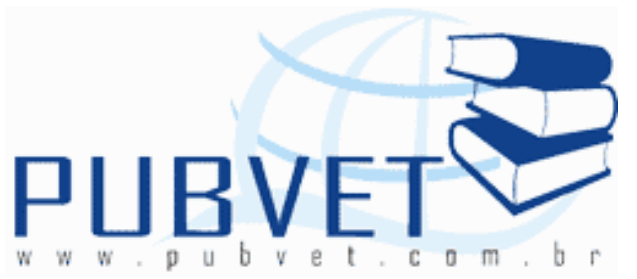

PUBVET, Publicações em Medicina Veterinária e Zootecnia.

\title{
Oxidação lipídica em filés de tambaqui (Colossoma macropomum) defumados com alecrim (Rosmarinus officinalis)
}

Fabíola Helena dos Santos Fogaça ${ }^{1}$, Sidely Gil Alves Vieira ${ }^{2}$, Thais Danyelle Santos Araújo², Luiz Gonzaga Alves dos Santos-Filho², João Avelar Magalhães³, Newton de Lucena Costa ${ }^{4}$

${ }^{1}$ Zootecnista, D.Sc., Pesquisador da Embrapa Meio-Norte, Parnaíba, Piauí.

${ }^{2}$ Estudante de Engenharia de Pesca da UFPI, Parnaíba, Piauí.

${ }^{3}$ Méd.Vet., D.Sc., Pesquisador da Embrapa Meio-Norte, Parnaíba, Piauí.

${ }^{4}$ Eng. Agr., D.Sc. Pesquisador da Embrapa Roraima, Boa Vista, Roraima.

\section{Resumo}

O tambaqui (Colossoma macropomum) é a espécie de peixe nativo de água doce mais produzida no Brasil. Sua carne apresenta excelente sabor e suculência, devido à presença de altos teores de lipídeos em sua composição. Essa característica torna seus filés mais suscetíveis às reações oxidativas que deterioram os lipídeos, produzindo sabor e odor de ranço. Compostos naturais com propriedades antioxidantes como o alecrim (Rosmarinus officinalis) são utilizados na prevenção da oxidação. O objetivo deste trabalho foi avaliar o efeito da adição de diferentes níveis de alecrim na oxidação lipídica e composição de filés defumados de tambaqui armazenados a $10^{\circ} \mathrm{C}$. Foi utilizado delineamento inteiramente casualizado, com seis níveis de adição de alecrim (zero; $0,1 \% ; 0,2 \%, 0,4 \%, 0,8 \%$ e $1,6 \% / \mathrm{kg}$ de filé) e dois tempos de avaliação 
FOGAÇA, F.H.S. et al. Oxidação lipídica em filés de tambaqui (Colossoma macropomum) defumados com alecrim (Rosmarinus officinalis). PUBVET, Londrina, V. 8, N. 10, Ed. 259, Art. 1717, Maio, 2014.

(I e II) para oxidação. Os filés foram submetidos a uma salmoura contendo o alecrim, conforme os tratamentos citados. Os filés foram salgados por 20 minutos e defumados por 4 horas. A composição foi determinada por metodologia analítica padrão e a oxidação lipídica, pela formação de substâncias reativas ao ácido tiobarbitúrico (SRATB), sendo o resultado expresso em $\mathrm{mg}$ de malonaldeído/kg de filé, segundo a curva padrão $\mathrm{y}=$ $0,272 x-0,200\left(R^{2}=0,991\right)$. Houve incremento significativo na umidade $(P<0,05)$ causado pela adição do alecrim, enquanto os teores de lipídeos e os valores de SRATB não apresentaram diferenças $(P>0,05)$ entre os tratamentos. Conclui-se que a adição de alecrim comercial à salmoura antes da defumação, até o nível de 1,6\%, não se mostrou eficiente no controle da oxidação lipídica em filés de tambaqui. Outros níveis devem ser testados para determinação de um valor ideal.

Palavras-chave: antioxidantes, lipídeos, salmoura.

\section{Lipid oxidation on tambaqui (Colossoma macropomum) fillets smoked with rosemary (Rosmarinus officinalis)}

\section{Abstract}

The tambaqui (Colossoma macropomum) is a higher produced native species of fish in Brazil. His flesh has excellent flavor and juiciness due to the presence of high levels of lipids in their composition. This characteristic makes their fillets more susceptible to oxidative reactions that damage lipids, producing taste and odor of rancidity. Natural compounds with antioxidant properties like rosemary (Rosmarinus officinalis) are used to prevent oxidation. The aim of this study was to evaluate the effect of different levels of rosemary addition on lipid oxidation and composition of smoked fillets of tambaqui stored at $10^{\circ} \mathrm{C}$. A completely randomized design with six levels of rosemary addition (zero, $0.1 \%, 0.2 \%, 0.4 \%, 0.8 \%$ and $1.6 \% / \mathrm{kg}$ of fillet) and two times of lipid oxidation evaluation (I and II) was used. The fillets were subjected to a brine containing rosemary, as treatments. The fillets were salted for 20 minutes and 
FOGAÇA, F.H.S. et al. Oxidação lipídica em filés de tambaqui (Colossoma macropomum) defumados com alecrim (Rosmarinus officinalis). PUBVET, Londrina, V. 8, N. 10, Ed. 259, Art. 1717, Maio, 2014.

smoked during 4 hours. The composition was determined by standard methodology and lipid oxidation by thiobarbituric acid reactive substances (TBARS), the results were expressed as $\mathrm{mg}$ of malonaldehyde / $\mathrm{kg}$ of fillet, according to the standard curve $y=0.272 x-0.200\left(R^{2}=0.991\right)$. There was significant increase in moisture $(P<0.05)$ caused by the addition of rosemary, while the levels of lipids and TBARS values did not differ $(P>0.05)$ between treatments. It concludes that the addition of rosemary in brine before smoking, until $1.6 \%$ level was not effective in controlling lipid oxidation in fillets of tambaqui. Other levels should be tested to determine an optimal value.

Keywords: antioxidants, brine, lipids.

\section{Introdução}

O Brasil possui o maior número de espécies de peixes de água doce do mundo e é detentor de aproximadamente $12 \%$ da água doce disponível no planeta, porém, por razões econômicas e culturais, tem sua piscicultura continental constituída principalmente pelo cultivo de espécies exóticas (FAO, 2006). No entanto esse cenário tende a se modificar, considerando-se os resultados de pesquisas realizadas com espécies nativas, principalmente aquelas com boa aceitação pelo mercado interno. Entre essas espécies, está o tambaqui (Colossoma macropomum), que apresenta rápido crescimento, boa aceitação de rações e carne com excelente sabor e composição nutricional (Cartonilho \& Jesus, 2011).

O consumo brasileiro de peixes ainda é baixo, em parte em razão de a comercialização do produto ser baseada no pescado inteiro eviscerado (Valenti et al., 2000). Por isso, a importância de se desenvolverem produtos préprontos, com sabores diferenciados e mais atrativos. A defumação é um método tradicional de conservação, pois combina os efeitos da salga, cocção, secagem e deposição de substâncias químicas bacteriostáticas da fumaça (fenóis, aldeídos e ácidos orgânicos) (Souza, 2003). Atualmente, é utilizada para proporcionar ao pescado características organolépticas desejáveis, como 
FOGAÇA, F.H.S. et al. Oxidação lipídica em filés de tambaqui (Colossoma macropomum) defumados com alecrim (Rosmarinus officinalis). PUBVET, Londrina, V. 8, N. 10, Ed. 259, Art. 1717, Maio, 2014.

cor, aroma, sabor e textura (Santos et al., 2007), sendo apreciada pelos consumidores.

Além dos métodos convencionais de conservação, existem substâncias naturais utilizadas na preservação do pescado, que conferem sabor aos alimentos e também possuem propriedades antimicrobianas e antioxidantes (Shelef, 1983). Essas substâncias antioxidantes podem ser usadas durante a elaboração de produtos, no intuito de estender sua vida de prateleira, porque seus compostos ativos ligam-se às moléculas de lipídeos, protegendo a membrana celular da oxidação causada por radicais livres presentes no próprio músculo (Devlin, 1997).

Larosa et al. (2012) avaliaram o efeito antioxidante do uso de orégano, alecrim, sálvia e moringa na conservação de carne mecanicamente separada (CMS) de tilápia (Oreochromis niloticus). Os resultados mostraram maior efeito antioxidante da sálvia na CMS de tilápia durante 120 dias de armazenagem a $-18^{\circ} \mathrm{C}$. Em outro estudo, o alecrim mostrou-se eficiente na conservação da CMS de sardinha (Sardina pilchardus) durante cinco meses de armazenagem a $-18^{\circ} \mathrm{C}$ (Serdarolu \& Felekolu, 2005). Em filés salgados de tilápia, houve um efeito protetor do alecrim (Afonso \& Sant'Ana, 2008). Recentemente, excelentes resultados foram obtidos com o uso de alecrim na conservação do camarão branco (Litopenaeus vannamei) descascado e congelado, que apresentou estabilidade lipídica e manutenção de suas características organolépticas durante 120 dias de armazenagem a $-18^{\circ} \mathrm{C}$ (Seabra et al., 2011).

Assim, o objetivo do estudo foi avaliar o efeito antioxidante da adição do alecrim em filés de tambaqui defumados, armazenados a $10^{\circ} \mathrm{C}$ durante sete dias.

\section{Material e Métodos}

O experimento foi conduzido no Laboratório de Análise e Processamento de Alimentos da Embrapa Meio-Norte, Parnaíba, Piauí. Foram utilizados 18 tambaquis, capturados de viveiros da Estação de Piscicultura do DNOCS 
FOGAÇA, F.H.S. et al. Oxidação lipídica em filés de tambaqui (Colossoma macropomum) defumados com alecrim (Rosmarinus officinalis). PUBVET, Londrina, V. 8, N. 10, Ed. 259, Art. 1717, Maio, 2014.

(Departamento Nacional de Obras Contra as Secas), no município de Piripiri, Piauí, insensibilizados com choque térmico em caixas isotérmicas contendo gelo em escama e água ( $1: 1 \mathrm{~kg} / \mathrm{L})$. Após o abate, foram transportados à Embrapa, em caixas térmicas contendo gelo. No laboratório foram pesados em balança digital (Shimadzu, modelo BL 3200 H, Japão), medidos em ictiômetro para se obter o comprimento padrão (extremidade cranial até o início da nadadeira caudal), eviscerados e cortados em duas partes ou bandas com o auxílio de uma serra-fita de coluna (1 HP, lâmina 218, modelo MA 680, Marconi, Campinas, Brasil). Os peixes apresentaram peso e comprimento médios de $1,47 \pm 0,24 \mathrm{~kg}$ e $36,29 \pm 2,26 \mathrm{~cm}$ respectivamente.

Foi utilizado o delineamento inteiramente casualizado com seis níveis de adição de alecrim (zero; $0,1 \% ; 0,2 \%, 0,4 \%, 0,8 \%$ e 1,6\%/kg de filé) e dois tempos de avaliação, com três repetições cada, sendo um filé considerado uma unidade experimental. Os filés foram separados em seis lotes, cada um correspondendo a um tratamento. Cada filé foi pesado e identificado com uma placa de polietileno.

Para defumação, os filés foram submetidos a uma salmoura a $20 \% \mathrm{p} / \mathrm{v}$, na proporção de 2:1 (volume da salmoura em litros/kg filés). O alecrim foi desidratado em estufa a $50^{\circ} \mathrm{C}$ (SOLAB, Piracicaba, Brasil), triturado manualmente em partículas de $1,0 \mathrm{~mm}$ de diâmetro aproximadamente e adicionado à salmoura, conforme os tratamentos. Os filés foram salgados por 20 minutos, drenados por 1 hora, distribuídos em grelhas perfuradas e colocados no defumador para desidratação parcial com aquecimento a gás, a $50^{\circ} \mathrm{C}$ por 60 minutos. Decorrido esse período, iniciou-se a adição da fumaça pela queima da serragem, a $60^{\circ} \mathrm{C}$ por 4 horas (Santos et al., 2007).

A defumação foi realizada em defumador industrial (Metalúrgica Lisboa Ltda., Minas Gerais, Brasil), com geração de fumaça fora da câmara de defumação. A serragem utilizada na produção de fumaça foi de pau d'arco ou ipê amarelo (Tabebuia serratifolia). Utilizou-se gás de cozinha (butano) para a manutenção da temperatura. 
FOGAÇA, F.H.S. et al. Oxidação lipídica em filés de tambaqui (Colossoma macropomum) defumados com alecrim (Rosmarinus officinalis). PUBVET, Londrina, V. 8, N. 10, Ed. 259, Art. 1717, Maio, 2014.

Após a defumação, os filés foram embalados em sacos de polietileno, identificados e armazenados a $10^{\circ} \mathrm{C}$ para as análises no tempo I (um dia de refrigeração) e tempo II (sete dias de refrigeração).

Para as análises químicas, foram retiradas amostras da musculatura lombar de cada filé, homogeneizadas e armazenadas a $10^{\circ} \mathrm{C}$. A umidade e o lipídeo foram determinados segundo método analítico padrão (AOAC, 2005). Foram analisados três filés de cada tratamento, em triplicata. A umidade foi determinada por diferença de peso de uma amostra de 2,0 g antes e após secagem em estufa (SOLAB, Piracicaba, Brasil) por 16 horas, a $105^{\circ} \mathrm{C}$. Os lipídeos foram determinados por extração em éter de petróleo em aparelho Sohxlet. A oxidação lipídica foi determinada pela formação de substâncias reativas ao ácido tiobarbitúrico (SRATB), sendo o resultado expresso em $\mathrm{mg}$ de malonaldeído/kg de filé, utilizando-se a equação da curva padrão y $=0,272 x-$ $0,200\left(R^{2}=0,991\right)$, segundo método de Vyncke (1970). As análises foram realizadas nos tempos I e II, correspondendo a um e sete dias de armazenamento refrigerado $\left(10^{\circ} \mathrm{C}\right.$ em geladeira). Foram realizadas leituras em comprimentos de onda de $400 \mathrm{~nm}$ em espectrofotômetro ( $\mathrm{HACH}$, mod. 2800, Alemanha), para quantificar os compostos secundários da oxidação produzidos abaixo de $500 \mathrm{~nm}$ (Berset \& Cuvelier, 1996).

Os dados obtidos foram analisados pelo programa estatístico ASSISTAT, versão 7.7 beta (2011). As médias foram comparadas pelo teste de Tukey ao nível de $5 \%$ de probabilidade.

\section{Resultados e Discussão}

Os teores de umidade e lipídeos determinados nos filés de tambaqui defumados estão descritos na Tabela 1. Nota-se, pela função da regressão quadrática, que houve incremento significativo na umidade $(P<0,05)$ causado pela adição do alecrim, enquanto os teores de lipídeos não variaram entre os tratamentos testados. Os menores teores de umidade foram determinados nos tratamentos controle e contendo $0,1 \%$ de alecrim. Houve incremento de $20,25 \%$ nos teores de umidade no tratamento com $0,2 \%$ de alecrim. 
FOGAÇA, F.H.S. et al. Oxidação lipídica em filés de tambaqui (Colossoma macropomum) defumados com alecrim (Rosmarinus officinalis). PUBVET, Londrina, V. 8, N. 10, Ed. 259, Art. 1717, Maio, 2014.

$\mathrm{Na}$ literatura não existe relação entre teores maiores ou menores de umidade e o uso de alecrim. Porém pode haver um efeito antioxidante da adição do alecrim na prevenção de danos celulares nos filés, prevenindo a perda de água durante a defumação, como observado em estudos utilizando a vitamina $\mathrm{E}$ na manutenção da qualidade de filés de salmão (Salmo salar L.) (Hang, 2011).

Tabela 1. Teores de umidade (\%) e lipídeo (\%) dos filés de tambaqui defumados.

\begin{tabular}{ccc}
\hline Tratamento & Umidade $(\%)$ & Lipídeo (\%) \\
\hline Controle & $68,99^{\mathrm{c}}$ & $0,24 \mathrm{a}$ \\
$0,1 \%$ & $71,70^{\mathrm{c}}$ & $0,18^{\mathrm{a}}$ \\
$0,2 \%$ & $89,15^{\mathrm{a}}$ & $0,21^{\mathrm{a}}$ \\
$0,4 \%$ & $75,30^{\mathrm{b}}$ & $0,32 \mathrm{a}$ \\
$0,8 \%$ & $75,97^{\mathrm{b}}$ & $0,29 \mathrm{a}$ \\
$1,6 \%$ & $76,49^{\mathrm{b}}$ & $0,15 \mathrm{a}$ \\
\hline Média & 76,17 & 0,23 \\
\hline CV (\%) & 2,94 & Sem ajustes \\
\hline Regressão & 0,999 & \\
\hline $\mathrm{R}^{2}$ & $\mathrm{y}=68,99-255,34 \mathrm{x}+3550,25 \mathrm{x}^{2}$ & \\
\hline
\end{tabular}

Médias seguidas de mesma letra não diferem entre si $(P>0,05)$ pelo Teste de Tukey.

Os resultados de oxidação lipídica determinados nos filés de tambaqui defumados com ou sem alecrim não apresentaram diferença $(P>0,05)$ entre os tratamentos ou nos tempos de avaliação (Tabela 2).

Observou-se que o efeito antioxidante do alecrim não é de rápida ação, pois logo após a aplicação do tratamento não houve redução dos valores de SRATB dos filés. No entanto, após sete dias de armazenamento refrigerado (Tempo II), a regressão obteve uma curva quadrática que indicaria uma função linear do efeito antioxidante do alecrim até o nível de 0,8\% e uma função quadrática em níveis iguais ou maiores a 1,6\%, com provável redução da oxidação ao longo do tempo (Tabela 2).

Esse efeito foi observado em filés salgados e secos de tilápia congelados durante 240 dias. Nos primeiros tempos de avaliação, não houve redução dos valores de SRATB, porém, com 240 dias, o valor de SRATB nos filés tratados com alecrim foi $50 \%$ menor quando comparado ao controle (Afonso \& Sant'Ana, 2008). 
FOGAÇA, F.H.S. et al. Oxidação lipídica em filés de tambaqui (Colossoma macropomum) defumados com alecrim (Rosmarinus officinalis). PUBVET, Londrina, V. 8, N. 10, Ed. 259, Art. 1717, Maio, 2014.

Tabela 2. Valores de SRATB em filés de tambaqui defumados.

\begin{tabular}{|c|c|c|c|c|c|c|c|c|c|c|}
\hline $\begin{array}{c}\text { Tratam } \\
\text { entos }\end{array}$ & Controle & 0,1 & 0,2 & $\frac{0,4}{\%}$ & 0,8 & 1,6 & Média & $\begin{array}{l}\text { CV } \\
(\%)\end{array}$ & Regressão & $\begin{array}{l}\mathrm{R}^{2} \\
(\%)\end{array}$ \\
\hline $\begin{array}{c}\text { Tempo } \\
\text { I }\end{array}$ & 1,05 & 1,12 & 1,14 & 1,20 & 0,92 & 1,05 & $1,008^{A}$ & - & $\begin{array}{c}\text { Sem } \\
\text { ajuste }\end{array}$ & - \\
\hline $\begin{array}{c}\text { Tempo } \\
\text { II }\end{array}$ & 1,30 & 1,03 & 1,12 & 0,76 & 0,84 & 0,75 & $0,970^{A}$ & 14,5 & $\begin{array}{c}y=0,398 \\
-0,918 x \\
+1,219 x^{2}\end{array}$ & 78 \\
\hline Média & $1,18^{\mathrm{a}}$ & $1,08^{a}$ & $1,14^{\mathrm{a}}$ & $0,98^{a}$ & $0,88^{a}$ & $0,90^{a}$ & & & & \\
\hline CV (\%) & & & & 17,2 & & & & & & \\
\hline
\end{tabular}

Médias seguidas de mesma letra, maiúsculas nas colunas e minúsculas nas linhas, não diferem entre si $(P>0,05)$ pelo Teste de Tukey. Tempo I: um dia de refrigeração; Tempo II: sete dias de refrigeração.

No caso dos filés de tambaqui, o valor de SRATB do grupo contendo $1,6 \%$ de alecrim no tempo II foi $43 \%$ menor quando comparado ao grupo controle, porém, estatisticamente, essa diferença não foi representativa. Em sardinhas (Sardina pilchardus), também não houve diferença $(P>0,05)$ nos valores de SRATB entre o grupo controle e o tratado com extrato de alecrim nas análises no tempo zero e após 30 dias de armazenamento em $-18^{\circ} \mathrm{C}$ (Serdarolu \& Felekolu, 2005).

A utilização do alecrim durante a salga teve o propósito de inserir um sabor atrativo ao produto defumado, sem alterar o método de defumação tradicional que utiliza a desidratação osmótica ou salga úmida como prétratamento do peixe (Agustinelli et al., 2013). A salmoura é um processo de transferência de massa em dois fluxos simultâneos e opostos: uma saída de água do produto para a solução e uma migração de solutos da solução para o produto (Ribeiro et al., 2010) que dependem da concentração de sal e outros compostos na solução utilizada (Sabadini et al., 2001). Em soluções binárias (sal + água), essa difusão é contínua, mas em soluções ternárias (sal + água + alecrim), há um menor fluxo de absorção dos solutos para o músculo (Medina-Vivanco et al., 2006), o que explicaria os valores semelhantes de SRATB entre os tratamentos pela baixa absorção de alecrim pelo filé (Tabela 2).

Existem muitas substâncias produzidas por reações oxidativas em alimentos. Cada uma é avaliada por absorbância em diferentes comprimentos 
FOGAÇA, F.H.S. et al. Oxidação lipídica em filés de tambaqui (Colossoma macropomum) defumados com alecrim (Rosmarinus officinalis). PUBVET, Londrina, V. 8, N. 10, Ed. 259, Art. 1717, Maio, 2014.

de onda. Para oxidação lipídica, quantificam-se os peróxidos que oxidam na presença de $\mathrm{Fe}^{2+}$ a $\mathrm{Fe}^{3+}$ dosado por colorimetria sob a forma de cloreto ou tiocianato férrico em comprimentos de onda $K<500 \mathrm{~nm}$ (Adams, 1995). Porém, por serem os peróxidos produtos primários da oxidação, são instáveis, decompondo-se em aldeídos, cetonas, hidroxiácidos, hidrocarbonetos e polímeros, designados como produtos secundários (Silva et al., 1999). Nesse sentido, a quantificação da oxidação é feita pela determinação do total de SRATB ou malonaldeídos em absorbâncias de 532 nm a 535 nm, mas há também absorbância de compostos secundários em absorbâncias próximas a 400 nm (Berset \& Cuvelier, 1996).

A formação desses compostos secundários em comprimentos de onda de $400 \mathrm{~nm}$ foi observada em filés de tambaqui nos tempos I e II (Figura 1) de avaliação. Porém não houve diferença significativa entre os tratamentos $(P>0,05)$, indicando que a presença do alecrim nos níveis testados $(0,1 \%$ a $1,6 \%)$ não foi suficiente para reduzir a oxidação em filés de tambaqui defumados. Esses resultados também foram observados em filés crus e cozidos de sardinha (Sardinella pilchardus) tratados com 1,0\% de extrato de alecrim, que tiveram boa aceitação na análise sensorial, no entanto, para os parâmetros bioquímicos (SRATB e valores de peróxido), apenas níveis de 2,0\% de alecrim combinados com embalagens a vácuo foram efetivos no controle da taxa de oxidação lipídica (Ozogul et al., 2010).

\section{Conclusões}

A adição de alecrim comercial à salmoura antes da defumação, até o nível de $1,6 \%$, não é eficiente no controle da oxidação lipídica em filés de tambaqui defumados armazenados por um período de sete dias, a $10^{\circ} \mathrm{C}$. Outros estudos para avaliação de níveis maiores de inclusão, bem como tempos mais extensos de prateleira dos produtos, devem ser realizados para que o alecrim seja eficiente na preservação de filés de tambaqui. 
FOGAÇA, F.H.S. et al. Oxidação lipídica em filés de tambaqui (Colossoma macropomum) defumados com alecrim (Rosmarinus officinalis). PUBVET, Londrina, V. 8, N. 10, Ed. 259, Art. 1717, Maio, 2014.

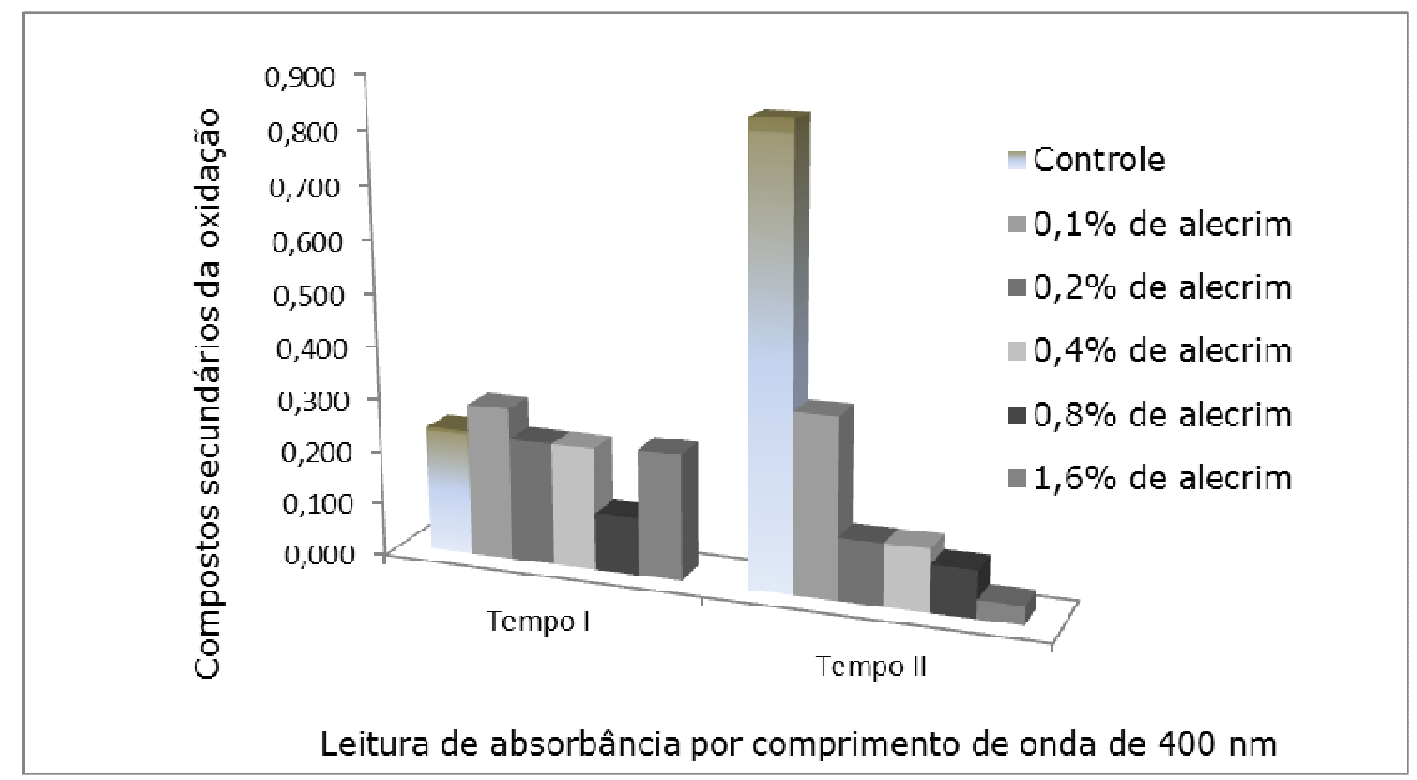

Figura 1. Determinação de compostos secundários a $400 \mathrm{~nm}$, nos tempos I (um dia de refrigeração) e II (sete dias de refrigeração).

\section{Agradecimentos}

Ao Departamento Nacional de Obras Contra as Secas - DNOCS, Estação de Piscicultura de Piripiri, PI, pela doação dos peixes utilizados no presente estudo.

\section{Referências Bibliográficas}

ADAMS, P.E. Determining iron content in foods by spectrophotometry. Journal of Chemical Education, v.72, n.7, p.649, 1995.

AFONSO, M. da S.; SANT'ANA, L.S. Effects of pretreatment with rosemary (Rosmarinus officinalis I.) in the prevention of lipid oxidation in salted tilapia fillets. Journal of Food Quality, v.31, n.1, p.586-595, 2008.

AOAC. Official methods of analysis of the Association of Official Analytical Chemists. 18th ed. Arlington: Association of Analytical Chemists, 2005.

ASSISTAT. Versão 7.7 beta Por Francisco de A. S. e Silva. DEAG-CTRN-UFCG - Atualizado em 04/03/2011. Disponível em <HTTP://www.assistat.com> Acesso em 01/06/2011.

AGUSTINELLI, S.P.; SALVADORI, V.O.; YEANNES, M.I. Determination of the moisture sorption behavior of osmotically dehydrated mackerel fillets by means ofbinary and ternary solutions. Food Science and Technology International, 2013, DOI: 10.1177/1082013213488610.

BERSET, C.; CUVELIER, M.E. Méthodes d'évaluation du degree d'oxydation des lipids et de mesure du pouvoir antioxidant. Sciences des Aliments, v.16, p.219-245, 1996. 
CARTONILHO, M.C.; JESUS, R.S. Qualidade de cortes congelados de tambaqui cultivado. Pesquisa Agropecuária Brasileira, v.46, n.4, p.344-350, abr., 2011.

DEVLIN, T.M. Manual de bioquímica com correlações clínicas. 4 ed. São Paulo: Edgard Blücher, 1997. 1007p.

FAO. State of world aquaculture. Rome: Food and Agricultural Organization of the United Nations. 2006. 145p. Disponível em<http://www.fao.org > Acesso em 01/10/2011.

HANG, A.T.N. Effects of dietary oxidation status and vitamin E level on performance, fillet quality and robustness to acute stress in Atlantic salmon (Salmo salar L.). 2011. 56fls. Dissertação (Mestrado em Aquicultura) - Universidade Norueguesa de Ciências da Vida, Noruega, 2011.

LAROSA, G.; CARVALHO, M.R.B.; VIDOTTI, R.M.; LIMA, T.M.A.; ALVES, V.F. Elaboração de produto cárneo de tilápia com antioxidantes visando sua utilização como recheio ou acompanhamento da refeição. Alimentos e Nutrição, v.23, n. 4, p. 609-617, out./dez, 2012.

MEDINA-VIVANCO, M.L.; SOBRAL, P.J. do A.; HUBINGER, M. D. Kinetic Aspects of Salting Tilapia Fillets Using Small Volumes of Brine. Brazilian Journal of Food and Technology, v.9, n.1, p.9-17, jan/mar, 2006.

OZOGUL, Y.; AYAS, D.; YAZGAN, H.; OZOGUL, F.; BOGA, E.K.; OZYURT, G. The capability of rosemary extract in preventing oxidation of fish lipid. International Journal of Food Science and Technology, v.45, p.1717-1723, 2010.

RIBEIRO, S.C.A.; PARK, K.J.; HUBINGER, M.D.; RIBEIRO, C.F.A.; ARAUJO, E.A.F.; TOBINAGA, S. Análise sensorial de músculo de mapará com e sem tratamento osmótico. Ciência e Tecnologia de Alimentos, v.30, n.1, p.24-32, maio, 2010.

SABADINI, E.; HUBINGER, M.D.; SOBRAL, P.J. do A.; CARVALHO, B.C. Alterações da atividade de água e da cor da carne no processo de elaboração da carne salgada desidratada. Ciência e Tecnologia de Alimentos, v.21, n.1, p.14-19, jan-abr, 2001.

SANTOS, L.D.; ZARA, R.F.; JESUÍ, V.V.; MATSUSHITA, M.; SOUZA, N.E.; SOUZA-FRANCO, M.L.R. Avaliação sensorial e rendimento de filés defumados de tilápia(Oreochromis niloticus Linnaeus, 1757) na presença de alecrim (Rosmarinus officinalis). Ciência Agrotécnica, v.31, n.2, p.406-412, mar./abr., 2007.

SEABRA, L.M.J.; DAMASCENO, K.S.F.S.C.; ANDRADE, S.A.C.; DANTAS, M.M.G.; SOARES, N.K.M.; PEDROSA, L.F.C. Effect of rosemary on the quality characteristics of white shrimp (Litopenaeus vannamei). Journal of Food Quality, v.34, n.1, p.363-369, 2011.

SERDAROLU, M.; FELEKOLU, E. Effects of using rosemary extract and onion juice on oxidative stability of sardine (Sardina pilchardus) mince. Journal of Food Quality, v.28, n.1, p.109120, 2005.

SHELEF, L.A. Antimicrobial effects of spices. Journal of Food Safety, v.6, p.29-44, 1983.

SILVA, F.A.M.; BORGES, M.F.M.; FERREIRA, M. Métodos para avaliação do grau de oxidação lipídica e da capacidade antioxidante. Química Nova, v.22, n.1, p.94-103, 1999.

SOUZA, M.L.R. Processamento do filé e da pele da tilápia do Nilo (Oreochromis niloticus): aspectos tecnológicos, composição centesimal, rendimento, vida útil do filé defumado e teste de resistência da pele curtida. 2003. 169f. Tese (Doutorado em Zootecnia) Universidade Estadual Paulista, Jaboticabal, 2003.

VALENTI, W.C.; POLI, C.R.; PEREIRA, J.A. Aquicultura no Brasil: bases para o desenvolvimento sustentável. 1 ed. Brasília: Ministério da Ciência e Tecnologia, 2000. 399p.

VYNCKE, W. Direct determination of the thiobarbituric acid value in trichloroacetic extracts of fish as a measure of oxidative rancidity. Feete-Scifen Anstrichmittel, v.72, n.12, p.10841087, 1970. 